\title{
XMM-Newton observations of HESS J1813-178 reveal a composite Supernova remnant
}

\author{
S. Funk ${ }^{1,2}$, J. A. Hinton ${ }^{3}$, Y. Moriguchi ${ }^{1,4}$, F. A. Aharonian ${ }^{5,1}$, Y. Fukui ${ }^{4}$, W. Hofmann ${ }^{1}$, D. Horns ${ }^{6}$, G. Pühlhofer ${ }^{7}$, \\ O. Reimer ${ }^{8}$, G. Rowell ${ }^{9}$, R. Terrier ${ }^{10}$, J. Vink ${ }^{11}$, and S. J. Wagner ${ }^{7}$
}

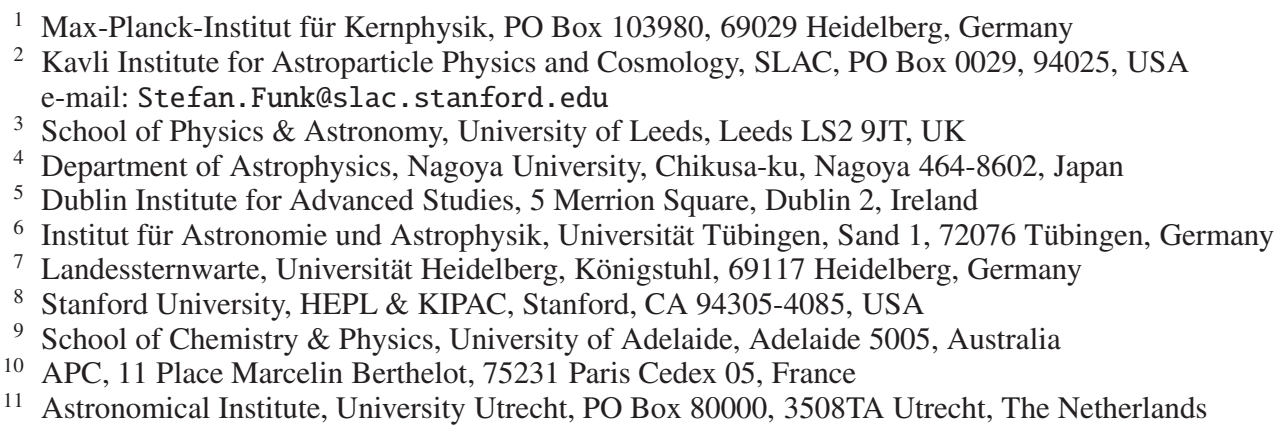

Received 20 November 2006 / Accepted 2 April 2007

\begin{abstract}
Aims. We present X-ray and ${ }^{12} \mathrm{CO}(J=1-0)$ observations of the very-high-energy (VHE) $\gamma$-ray source HESS J1813-178 with the aim of understanding the origin of the $\gamma$-ray emission.

Methods. High-angular resolution X-ray studies of the VHE $\gamma$-ray emission region are performed using 18.6 ks of XMM-Newton data, taken on HESS J1813-178 in October 2005. Using this data set we are able to undertake spectral and morphological studies of the X-ray emission from this object with greater precision than previous studies. NANTEN ${ }^{12} \mathrm{CO}(J=1-0)$ data are used to search for correlations of the $\gamma$-ray emission with molecular clouds which could act as target material for $\gamma$-ray production in a hadronic scenario.

Results. The NANTEN ${ }^{12} \mathrm{CO}(J=1-0)$ observations show a giant molecular cloud of mass $2.5 \times 10^{5} M_{\odot}$ at a distance of $4 \mathrm{kpc}$ in the vicinity of HESS J1813-178. Even though there is no direct positional coincidence, this giant cloud may have influenced the evolution of the $\gamma$-ray source and its surroundings. The X-ray data show a highly absorbed $\left(n_{\mathrm{H}} \sim 1 . \times 10^{23} \mathrm{~cm}^{-2}\right)$ non-thermal X-ray emitting object coincident with the previously known ASCA source AXJ1813-178 exhibiting a compact core and an extended tail towards the north-east, located in the centre of the radio shell-type Supernova remnant (SNR) G12.82-0.02. This central object shows morphological and spectral resemblance to a Pulsar Wind Nebula (PWN) and we therefore consider that this object is very likely to be a composite SNR. We discuss the scenario in which the $\gamma$-rays originate in the shell of the SNR, and that in which they originate in the central object, in terms of a time-dependent one-zone leptonic model. We demonstrate, that in order to connect the core X-ray emission to the VHE $\gamma$-ray emission electrons have to be accelerated to energies of at least $1 \mathrm{PeV}$.
\end{abstract}

Key words. ISM: supernova remnants - ISM: individual objects: HESS J1813-178 - ISM: individual objects: G12.82-0.02 ISM: individual objects: AX J1813-178 - gamma rays: observations

\section{Introduction}

During a survey of the Galactic plane in very high-energy (VHE) $\gamma$-rays using the atmospheric Cherenkov telescope system HESS more than a dozen new $>100 \mathrm{GeV} \gamma$-ray sources were found (Aharonian et al. 2005a, 2006a). Even though some of the new $\gamma$-ray sources can be identified with counterparts at other wavebands, for example the microquasar LS 5039 (Aharonian et al. 2005b, 2006b), most of them lack a compelling positional counterpart (Funk 2007). A firm counterpart identification for a $\gamma$-source at other wavebands requires not only a spatial association, but also a viable $\gamma$-ray emission mechanism and a consistent multi-frequency picture. X-ray observations with instruments such as XMM-Newton and Chandra combine high-angular-resolution and high-sensitivity observations with the possibility to trace relativistic electrons via their synchrotron emission. These instruments are thus ideally suited to the identification of VHE $\gamma$-ray objects. This article reports on multi-frequency observations of HESS J1813-178 with the XMM-Newton X-ray satellite. Dense molecular regions that could act as target material for hadronic interactions have been studied using the NANTEN CO-telescope to test for an origin of the $\gamma$-ray emission in the decay of neutral pions produced in proton-proton collisions.

HESS J1813-178 was in fact the first source discovered during the HESS survey, and was subsequently re-observed to increase the $\gamma$-ray signal to 340 events (with a statistical significance of $\sim 14$ standard deviations) from a total of $9.7 \mathrm{~h}$ of observations. Whilst many of the new sources found in the survey exhibit rather large intrinsic extensions, HESS J1813-178 has a compact nature and is only slightly extended (Gaussian width $\sigma=2.2^{\prime} \pm 0.4^{\prime}$ ) with respect to the point-spread function (PSF) of the HESS instrument. The flux of the source 
is $\left(14.2 \pm 1.1_{\text {stat }}\right) \times 10^{-12}$ photons $\mathrm{cm}^{-2} \mathrm{~s}^{-1}$ above $200 \mathrm{GeV}$ and the energy spectrum follows a power-law with photon index $2.09 \pm 0.08$. HESS J1813-178 is located in the Galactic plane at $18^{\mathrm{h}} 13^{\mathrm{m}} 38^{\mathrm{s}},-17^{\mathrm{d}} 50^{\prime} 33^{\prime \prime}$. HESS J1813-178 was subsequently also detected by the MAGIC Cherenkov telescope confirming the basic properties of the source (Albert et al. 2006).

At the time of the first publication of this source (Aharonian et al. 2005a), HESS J1813-178 was still considered as unidentified. However, subsequently to the HESS publication, the source was reported as being in compelling positional coincidence with several objects known in other energy bands (Brogan et al. 2005; Helfand et al. 2005; Ubertini et al. 2005). HESS J1813-178 coincides with the previously unpublished archival ASCA source AX J1813-178 in the 2-10 keV band (Brogan et al. 2005). This unresolved ASCA source with a rather hard photon index of $1.8 \pm 0.4$ and a flux of $7 \times 10^{-12} \mathrm{erg} \mathrm{cm}^{-2} \mathrm{~s}^{-1}(2-10 \mathrm{keV})$ was one of the few relatively bright sources detected in the ASCA Galactic Plane survey (Sugizaki et al. 2001). AX J1813-178 exhibits a highly absorbed $\left(n_{\mathrm{H}}=(1.1 \pm 0.2) \times 10^{23} \mathrm{~cm}^{-2}\right)$ nonthermal spectrum without indications of emission lines. The value of $n_{\mathrm{H}}$ is significantly larger than the total column density through the Galaxy in this direction, suggesting that the source is embedded in a dense environment. The unresolved hard X-ray source IGR J18135-1751 detected in the INTEGRAL Galactic plane survey in the $20-100 \mathrm{keV}$ energy band also lies in positional coincidence (Ubertini et al. 2005). The energy spectrum of IGR J18135-1751 is compatible with that of the ASCA source in terms of flux, but is softer, suggesting a spectral break in the X-ray spectrum around $10 \mathrm{keV}$. Recently this source has also been detected with the Swift X-Ray Telescope (XRT). The XRT data confirm the hard spectral index and the high column density towards the source that were found in the ASCA data (Landi et al. 2006).

The region surrounding HESS J1813-178 was also covered by observations in the $20 \mathrm{~cm}$ and $90 \mathrm{~cm}$ band by the VLA. These data show a faint non-thermal radio source (G12.82-0.02) visible at the position of HESS J1813-178 (Brogan et al. 2005; White et al. 2005). G12.82-0.02 lies in the projected vicinity (distance $\sim 15^{\prime}$ ) of the W 33 region. This region is known to contain ultra-compact HII regions (Churchwell 1990) and contains methanol, hydroxyl, and water masers and other tracers of recent star formation. While HESS ASCA, and INTEGRAL lack the spatial resolution to resolve this object, the angular resolution of the VLA allows a shell-like radio structure of diameter $2.5^{\prime}$ to be resolved. The flux at $20 \mathrm{~cm}$ was reported as $0.65 \pm 0.1 \mathrm{Jy}$, the flux at $90 \mathrm{~cm}$ was given as $1.2 \pm 0.08 \mathrm{Jy}$, suggesting a non-thermal radio spectrum of index $\alpha=-0.42 \pm 0.03\left(j_{v} \propto v^{\alpha}\right)$. The radio spectrum and morphology led Brogan et al. (2005) to the conclusion that the radio structure is a previously unknown young shell-type Supernova remnant (SNR). Spitzer Space Telescope data from the GLIMPSE survey at $8 \mu \mathrm{m}$ (Benjamin et al. 2003) show no signs of dust emission in positional coincidence with G12.82-0.02 a finding that might be expected, given that Reach et al. (2006) detected only 18 out of 95 known SNRs in the GLIMPSE survey.

In the Parkes multi-beam pulsar survey (PMBS) no pulsar close to G12.82-0.02 has been found thereby limiting the $1.4 \mathrm{GHz}$ flux density to a rather constraining level of $<0.2 \mathrm{mJy}$ (Manchester et al. 2001). Current constraints on the distance of the object have been derived from $\mathrm{HI}$ absorption data (Brogan et al. 2005) as well as from the strong absorption found in the X-ray data, both suggesting that AX J1813-178 is located behind W 33 at a distance larger than $4 \mathrm{kpc}$.
Assuming an association between HESS J1813-178 and the radio source G12.82-0.02, the question remains whether the thus far unresolved X-ray and $\gamma$-ray emission originate from the shell of the SNR, or rather from a Pulsar Wind Nebula (PWN) embedded within the shell. Brogan et al. (2005) concluded from the fact that the radio spectrum nearly directly extrapolates into the ASCA X-ray spectrum, that both the radio shell and the ASCA source should indeed be the same source and therefore that all the emission was connected to the radio shell.

This article reports on high-angular resolution X-ray observations performed with XMM-Newton with the goal of pinning down the origin of the high-energy X-ray and $\gamma$-ray emission. Another option for the $\gamma$-ray emission is the interaction of accelerated hadrons with dense molecular material. The nearest massive molecular cloud is that associated with the W 33 star forming region, located $\sim 10^{\prime}$ away from HESS J1813-178. Dense gas in the W 33 region could act as target material for the VHE $\gamma$-ray generation in hadronic interactions, therefore a study of the ${ }^{12} \mathrm{CO}(J=1-0)$ distribution performed during the NANTEN survey of the Galactic plane (Mizuno \& Fukui 2004) towards HESS J1813-178 is included.

\section{XMM-Newton observations of the region}

HESS J1813-178 was observed with XMM-Newton on the 14th of October 2005 for $18.6 \mathrm{ks}$. The instrument cameras (EPIC MOS1, MOS2, PN) were operated in full-frame mode with a medium filter to screen out optical and UV light. The data were processed with the XMM-Newton Science Analysis Software (SAS) version 7.0 as well as with the Extended Source Analysis Software package (XMM-ESAS) version 1.0 (Snowden et al. 2004). Standard data reduction and calibration procedures were applied to the data and the total data set amounts to $13.6 \mathrm{ks}$ after the observations have been cleaned of flaring background caused by soft protons. Figure 1 shows the resulting combined count map of the EMOS1 and EMOS2 detectors for three different energy bands (red: $0.5 \mathrm{keV}$ to $2 \mathrm{keV}$, green: $2 \mathrm{keV}$ to $4.5 \mathrm{keV}$, blue: $4.5 \mathrm{keV}$ to $10 \mathrm{keV}$ ) smoothed with a Gaussian kernel of 3 pixels width. The X-ray morphology does not resemble the shell-like emission seen in the radio. A compact X-ray source with an extended tail towards the northwest is visible at the centre of the EMOS-Cameras. This source is in positional coincidence with the previously reported ASCA source AX J1813-178. There is some contamination of soft photons in this data set. This had been seen before in the ASCA data and can be attributed to the bright, close-by $\left(\sim 0.7^{\circ}\right)$ low mass $\mathrm{X}$-ray binary GX $13+1$. To investigate the influence of the soft photon contamination in the background regions for the spectral studies, three separate methods were applied to estimate the background as discussed later in the text. The results for the different methods show good agreement, enhancing the confidence that the background level in the spectral studies was estimated correctly. There is however still the possibility of soft photon contamination in the source spectrum, especially at energies below $2 \mathrm{keV}$, since the source appears strongly absorbed below this energy.

For the source position fitting the contamination was avoided by using the source detection algorithm (emldetect) as described in Snowden et al. (2004) for energies above $4.5 \mathrm{keV}$ and for energies above $7.5 \mathrm{keV}$. Seven sources are detected in the band above $4.5 \mathrm{keV}$ which are not in the part of the field of view affected by the stray light (see Table 1), whereas above $7.5 \mathrm{keV}$ only a single X-ray source is detected. This source is extended and coincident with AX J1813-178 at a best-fit position $18^{\mathrm{h}} 13^{\mathrm{m}} 35.16^{\mathrm{s}}$, 

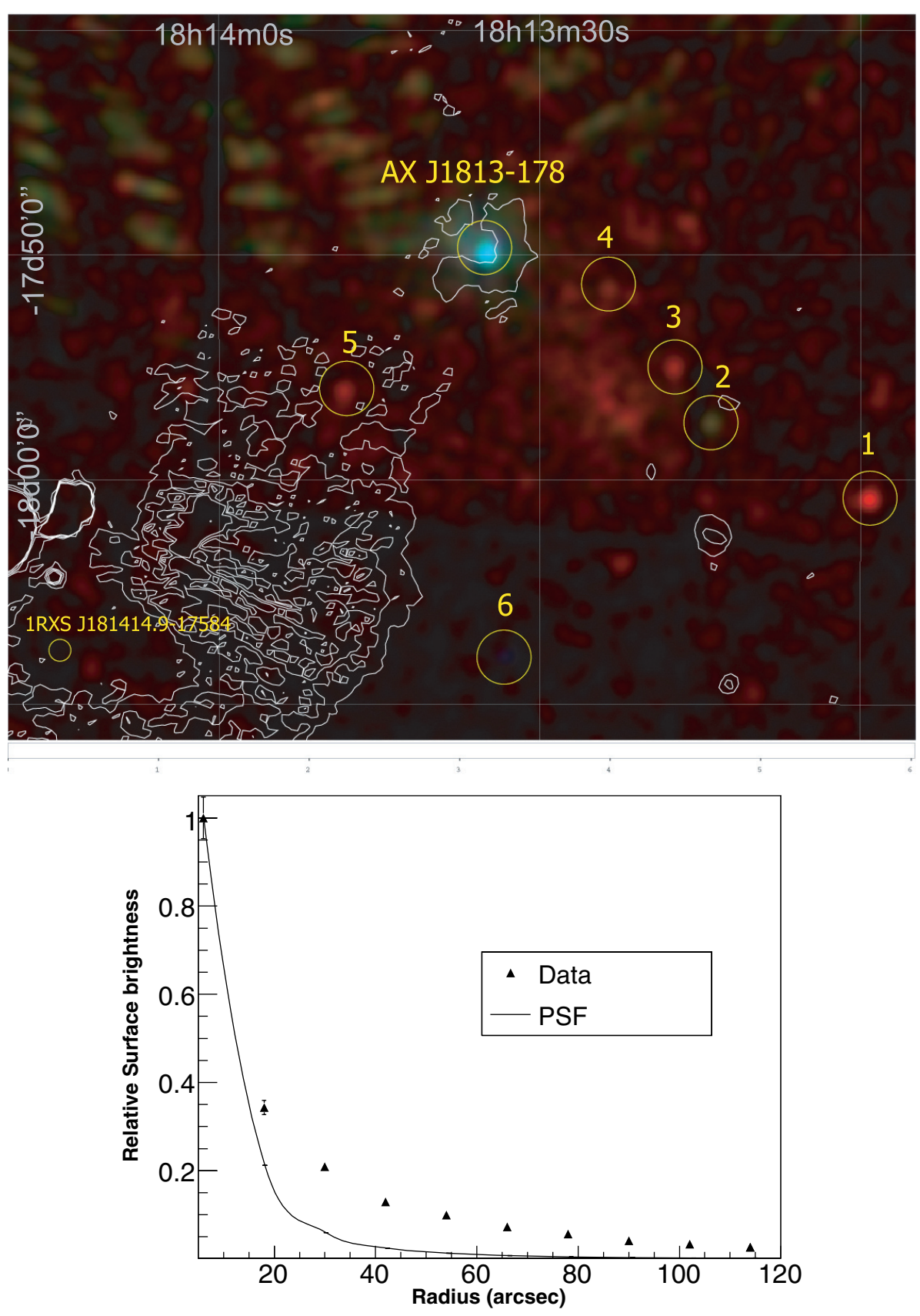

Fig. 1. Top: a composite false-colour image of the EMOS1 and EMOS2 count maps, slightly smoothed with a Gaussian kernel of width 3 image pixels. Different colours correspond to $0.5 \mathrm{keV}-2 \mathrm{keV}$ (red), $2 \mathrm{keV}-4.5 \mathrm{keV}$ (green), and $4.5 \mathrm{keV}-10 \mathrm{keV}$ (blue). AXJ1813-178 appears as a bright, hard-spectrum source with a tail towards the north-west. The northwestern edge of the field of view shows some soft stray-light contamination, caused by the low mass X-ray binary GX 13+1. Also shown are several other $\mathrm{X}$-ray sources that were found with emldetect at energies above $4.5 \mathrm{keV}$. These are typically fainter and have a softer photon index. The white contours denote the VLA $20 \mathrm{~cm}$ radio emission as already reported by Brogan et al. (2005). The shell-like radio structure surrounding AX J1813-178 is clearly visible. Bottom: radial profile of the central source AX J1813-178 in comparison to the PSF for this data set. $-17^{\mathrm{d}} 49^{\prime} 50.0^{\prime \prime}$, with a statistical error on the position of $2^{\prime \prime}$. This position is at a distance of $\sim 1^{\prime}$ from the best fit position of HESS J1813-178, located well within the extension of $2.2^{\prime}$ of HESS J1813-178. Even though a perfect match of the best fit $\mathrm{X}$-ray and $\gamma$-ray positions is not a priori expected, as seen for example in the PWN candidate HESS J1825-137 (in which the HESS position is significantly shifted from the peak of the Xray emission, Aharonian et al. 2006c) the position of AX J1813178 is compatible with the HESS position, given a statistical error of $21^{\prime \prime}$ adding to a systematic error in the pointing accuracy of the HESS instrument of the similar size. The source position fitting tool emldetect also determines AX J1813-178 to be incompatible with a point-source. Using a Gaussian model the extension was determined to be $8.5 \pm 0.2$ image pixels, corresponding to a projected width on the sky of $21^{\prime \prime} \pm 0.5^{\prime \prime}$. As apparent in the slice through the source, shown in Fig. 2, the Gaussian profile might not be the correct representation of the source profile, which seems to have a rather long tail towards the north-east. The source position determined using the XMMNewton data is compatible with the position of the only Xray source found in the Swift XRT data (Landi et al. 2006) at $18^{\mathrm{h}} 13^{\mathrm{m}} 34.9^{\mathrm{s}},-17^{\mathrm{d}} 49^{\prime} 53.2^{\prime \prime}$ with an error of $3.5^{\prime \prime}$. The distance of the best fit position presented here to the XRT position is $4.9^{\prime \prime}$. The other sources within the field of view listed in Table 1 have no previous X-ray detection.

For the spectral analysis XSPEC (version 12.2.1) was used and three different background methods were applied to estimate the effect of stray-light contamination on the spectrum of AXJ1813-178. The background was estimated a) from the south-western part of the field of view, where the stray-light contamination is apparently lower b) from a ring around the source with inner radius $100^{\prime \prime}$ and outer radius 200" and c) from blank field observations as described in Snowden et al. (2004). All three methods yield consistent results for the spectral fit. In the following the background derived from a ring around the source within the same field of view is used. Different extraction radii 
Table 1. X-ray sources other than AXJ1813-178 detected in the field of view surrounding HESS J1813-178 using the detection algorithm emldetect. The second column gives the name recommended by the $X M M-N e w t o n$ SOC and the IAU for serendipitous XMM-Newton source detections. Columns 3 and 4 give J2000 coordinates with a typical error on the position of $1^{\prime \prime}$. Column 5 gives the number of counts in a circle of radius $30^{\prime \prime}$ above $2 \mathrm{keV}$.

\begin{tabular}{ccccc}
\hline \hline Id & XMMUJ & $\begin{array}{c}\mathrm{RA}_{2000} \\
(\mathrm{~h}: \mathrm{m}: \mathrm{s})\end{array}$ & $\begin{array}{c}\text { Dec }_{2000} \\
\left(\mathrm{~d}::^{\prime \prime}\right)\end{array}$ & Counts \\
\hline 1 & $181259.2-175527$ & $18: 12: 59.23$ & $-17: 55: 26.9$ & 60 \\
2 & $181314.1-175344$ & $18: 13: 14.11$ & $-17: 53: 44.1$ & 238 \\
3 & $181318.0-175232$ & $18: 13: 17.57$ & $-17: 52: 31.9$ & 90 \\
4 & $181323.7-175041$ & $18: 13: 23.68$ & $-17: 50: 41.2$ & 138 \\
5 & $181348.3-175301$ & $18: 13: 48.35$ & $-17: 53: 00.6$ & 68 \\
6 & $181333.3-175857$ & $18: 13: 33.27$ & $-17: 58: 56.7$ & 157 \\
\hline & AXJ1813-178 & $18: 13: 35.16$ & $-17: 49: 50.0$ & 2192 \\
\hline
\end{tabular}

of size $50^{\prime \prime}, 75^{\prime \prime}$, and $100^{\prime \prime}$ were used to determine the spectrum of the extended emission. All EMOS1, EMOS2, and EPN data were fit simultaneously. In general the data are well described by a single power-law and the results of the spectral fitting are summarised in Table 2. Figure 3 shows the XMM-Newton spectrum for the medium size extraction radius of $75^{\prime \prime}$. As can be seen, no prominent line emission exists and analysis shows that the shape is incompatible with a black-body radiation spectrum, confirming the non-thermal nature of the X-ray emission. The results for the different extraction radii are entirely compatible with each other in terms of column density and spectral index. The column density determined is $n_{\mathrm{H}} \sim 10^{23} \mathrm{~cm}^{-2}$, significantly higher than the total Galactic column density in this region of the Galactic plane, $1.6 \times 10^{22} \mathrm{~cm}^{-2}$ (Dickey \& Lockman 1990). The photon index is determined to be $\sim 1.8$, the flux between 2 and $10 \mathrm{keV}$ for the $75^{\prime \prime}$ extraction radius is $\sim 7 \times 10^{-12} \mathrm{erg} \mathrm{cm}^{-2} \mathrm{~s}^{-1}$. Both these values are compatible with previous estimates from the analysis of ASCA data (photon index: $1.8 \pm 0.4, F_{2-10 \mathrm{keV}}=7.0 \times 10^{-12} \mathrm{erg} \mathrm{cm}^{-2} \mathrm{~s}^{-1}$ ) by Brogan et al. (2005). It should be noted that this flux possibly contains some contamination from a non-subtracted point-source. Any such contamination is expected to cover one or two bins in the slice shown in Fig. 2 and therefore amount to at most $20 \%$ of the flux.

Comparing the number of counts on the shell with those in the central source a conservative upper limit of the X-ray emission on the shell of $F_{2-10 \mathrm{keV}}=7.0 \times 10^{-12} \mathrm{erg} \mathrm{cm}^{-2} \mathrm{~s}^{-1}$ can be derived. Using a Raymond-Smith model (assuming thermal equilibrium between electron and ion plasma) this upper limit can be used to determine an upper limit on the emission measure $k=n_{\mathrm{e}} n_{\mathrm{H}} V\left(4 \pi D^{2}\right)^{-1}$ of an X-ray emitting thermal plasma under the assumption of various temperatures $T$ (here $n_{\mathrm{e}}$ and $n_{\mathrm{H}}$ correspond to the electron and hydrogen densities in the plasma, $V$ the volume of the plasma and $D$ the distance). For a temperature of $1 \mathrm{keV}$, the emission measure is $k=3.47 \times 10^{11} \mathrm{~cm}^{-5}$, corresponding to an upper limit on the density of $n_{\mathrm{H}}=4.1 \mathrm{~cm}^{-3}$ (at a distance of $4 \mathrm{kpc}$ ). Assuming a density of $n_{\mathrm{H}}=1 \mathrm{~cm}^{-3}$, the corresponding upper limit on the plasma temperature is $k T \sim 5 \mathrm{keV}$.

The EPN data within a narrow region $\left(5^{\prime \prime}\right)$ surrounding AX J1813-178 were searched for periodic emission from any possible X-ray pulsar. The timing resolution of the EPN detector in full-frame mode is $73.2 \mathrm{~ms}$. No significant periodicity was found. Indeed no evidence for variability was detected in this data set. Future X-ray timing observations with XMM-Newton or Chandra and future Multibeam radio observations might shed further light on the existence of a pulsar at this location.
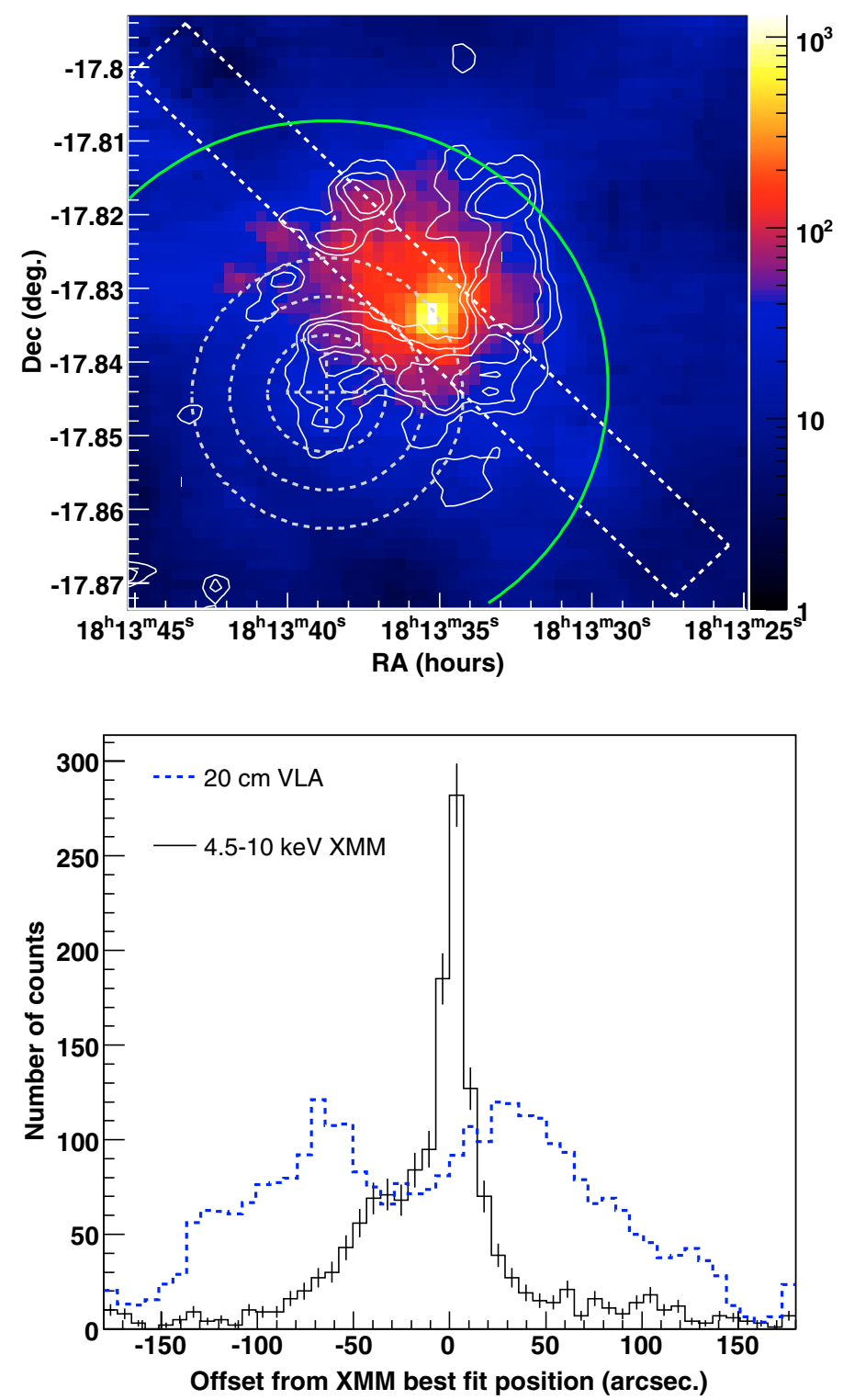

Fig. 2. Comparison of radio and X-ray data of HESS J1813-178. Top: XMM-Newton counts map above $4.5 \mathrm{keV}$ of the region surrounding HESS J1813-178 (colour contours) smoothed with a Gaussian kernel of width $0.1^{\prime}$. The extended tail towards the north-east is visible in this figure. Overlaid is the $20 \mathrm{~cm}$ shell-like emission (white contours) as detected by the VLA (Brogan et al. 2005). The difference in the images between the X-ray and the radio wavebands is apparent in this figure. Also shown are the positional contours (1,2,3 $\sigma$ error) of the best fit position of HESS J1813-178 as given in Aharonian et al. (2006a) (dashed circles) and the extension (solid green), covering both the radio and X-ray emitting region completely. Bottom: slice through the emission in radio and X-rays as plotted on the left hand side. The box in which the slices were determined is also given in the left panel (white box). The X-ray slice shows the compact core with the slice towards the north-east, whereas the radio slice shows the shell-like structure of the emission.

\section{NANTEN-observations towards HESS J1813-178}

The ${ }^{12} \mathrm{CO}(J=1-0)$ observations were performed using the 4-meter mm/sub-mm telescope, NANTEN, at Las Campanas Observatory of the Carnegie Institution of Washington, in Chile. The NANTEN ${ }^{12} \mathrm{CO}(J=1-0)$ survey of the Galactic plane shows that the velocity of the ${ }^{12} \mathrm{CO}$ emission towards HESS J1813-178 ranges from $v_{\mathrm{LSR}}$ (velocity local standard of 
Table 2. Spectral properties of AX J1813-178 as determined by the spectral fitting routine. EMOS1, EMOS2, and EPN data were simultaneously fit with a single absorbed power-law. The fit parameters are the column density $n_{\mathrm{H}}$, the photon index $\Gamma$, and the normalisation at $1 \mathrm{keV}$, which is expressed by the flux between $2 \mathrm{keV}$ and $10 \mathrm{keV}$. Different spectral shapes such as a black-body spectrum were incompatible with the data (as an example: the $\chi^{2} /$ d.o.f. for a blackbody spectrum for the extraction region of size $75^{\prime \prime}$ is 5700 . The errors given here correspond to a $90 \%$ confidence level.

\begin{tabular}{cccccccc}
\hline \hline $\begin{array}{c}\text { Radius } \\
\text { (arcsec) }\end{array}$ & EMOS1 & $\begin{array}{c}\text { Counts } \\
\text { EMOS2 }\end{array}$ & EPN & $\begin{array}{c}n_{\mathrm{H}} \\
10^{22} \mathrm{~cm}^{-3}\end{array}$ & $\Gamma$ & $\begin{array}{c}F_{2-10 \mathrm{keV}} \\
10^{-12} \mathrm{erg} \mathrm{cm}^{-2} \mathrm{~s}^{-1}\end{array}$ & $\chi^{2} /$ d.o.f. \\
\hline 50 & 2004 & 1805 & 3930 & $12.0_{-0.7}^{+1.0}$ & $1.81_{-0.11}^{+0.16}$ & 5.2 & $3177 / 3001$ \\
75 & 2957 & 2769 & 6194 & $10.6_{-0.7}^{+0.7}$ & $1.72_{-0.12}^{+0.12}$ & 6.8 & $3233 / 3001$ \\
100 & 3794 & 3592 & 8397 & $10.6_{-0.3}^{+0.2}$ & $1.88_{-0.03}^{+0.05}$ & 6.9 & $3228 / 3001$ \\
\hline
\end{tabular}

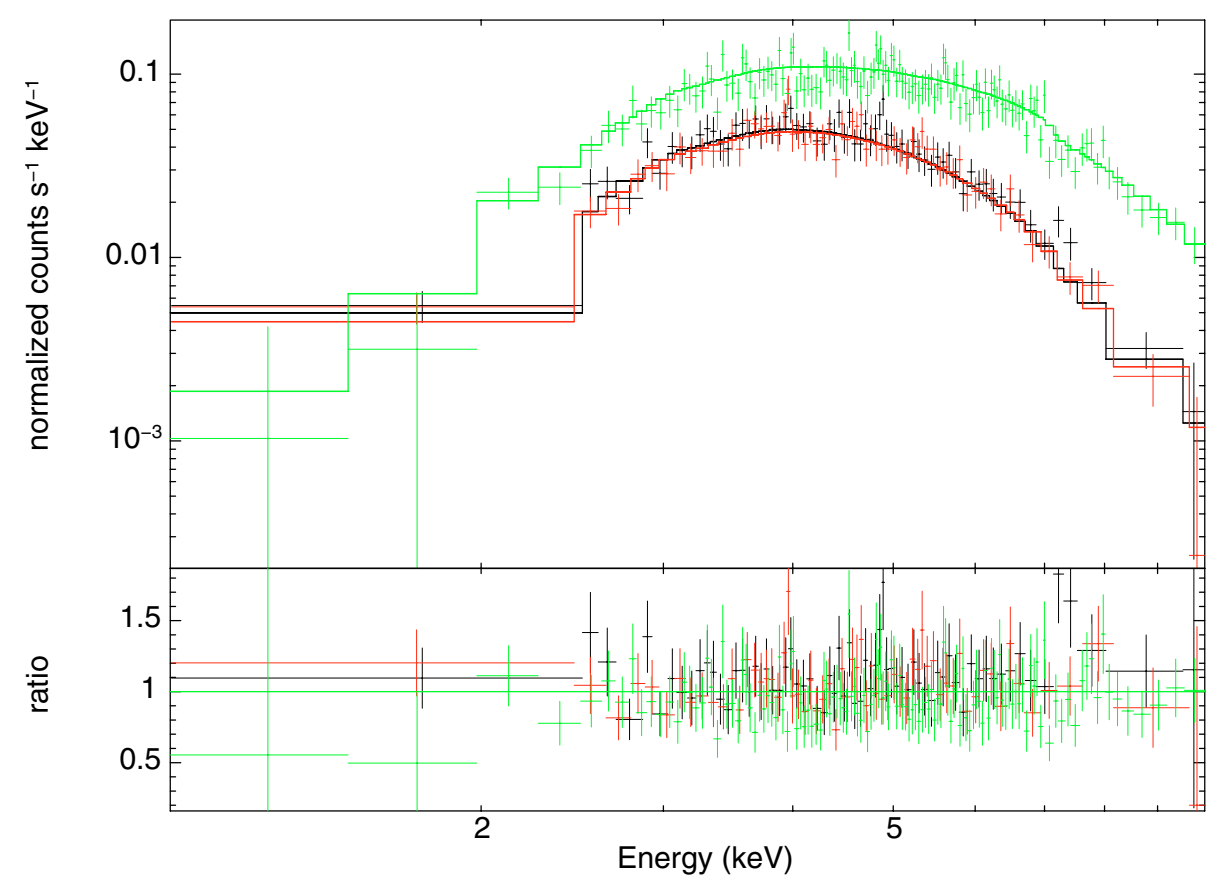

Fig. 3. XMM-Newton energy spectrum for all three detectors (EMOS1: black, EMOS2: red, EPN: green) for an extraction radius of $75^{\prime \prime}$, rebinned to yield a minimum significance of $5 \sigma$ in each bin, summing a maximum of 100 bins into a single one bin. The straight lines show the combined fit to these data (folded with the instrumental response which is different for the three detectors). The lower panel shows the residuals of the data to the fit. As can be seen, the power-law fit provides a reasonable description of the data. Also apparent is the absence of prominent line emission that lends support to the nonthermal nature of the emission.

rest, i.e. the average velocity of stars in the solar neighbourhood) $\sim 0 \mathrm{~km} \mathrm{~s}^{-1}$ to $\sim 60 \mathrm{~km} \mathrm{~s}^{-1}$. While the $\mathrm{CO}$ emission at $v_{\text {LSR }}$ less than $10 \mathrm{~km} \mathrm{~s}^{-1}$ is likely due to local clouds, the emission at velocities in excess of $v_{\text {LSR }} \sim 20 \mathrm{~km} \mathrm{~s}^{-1}$ is likely from clouds beyond $2 \mathrm{kpc}$. The best known object in the region surrounding HESS J1813-178 is W 33, a complex of HII regions, a typical example of a massive star forming region. The integrated intensity distribution at $v_{\mathrm{LSR}}=30-40 \mathrm{~km} \mathrm{~s}^{-1}$ shows an enhancement in close projected vicinity to HESS J1813-178 as shown in Fig. 4. To convert the velocity range into a distance the model of Brand \& Blitz (1993) has been used and the rather large uncertainty in kinematic distance for directions close to the Galactic centre has to be taken into account. The W 33 complex has been studied fairly extensively in the past and its distance has been estimated to be $\sim 4 \mathrm{kpc}$ (kinematic distance derived from molecular line observations see e.g. Reifenstein et al. 1970; Goldsmith \& Mao 1983; Mitchell et al. 1990). The most massive molecular cloud in the direction of HESS J1813-178 is in the velocity range $30-40 \mathrm{~km} \mathrm{~s}^{-1}$ as shown in Fig. 4. The size of this cloud is $\sim 70 \mathrm{pc} \times 40 \mathrm{pc}$ in Galactic longitude and latitude (at an assumed distance of $4 \mathrm{kpc})$ and its total mass is estimated to be $2.5 \times 10^{5}$ solar masses from the ${ }^{12} \mathrm{CO}(J=1-0)$ velocity integrated intensity, equivalent to $10^{21} \mathrm{~cm}^{-2}$ in molecular column density, assuming an $X$-factor of $2 \times 10^{20} \mathrm{~cm}^{-2} /\left(\mathrm{K} \mathrm{km} \mathrm{s}^{-1}\right)$ (Bertsch et al. 1993) (where $X=N\left(\mathrm{H}_{2}\right) / I(\mathrm{CO})$, is the conversion factor between $\mathrm{H}_{2}$ column density and integrated $\mathrm{CO}$ intensity). This mass is typical for a giant molecular cloud. HESS J1813-178 is clearly located outside of the dense cloud ( 20 pc away) and therefore the cloud and the VHE $\gamma$-ray emission are probably unrelated. The region surrounding HESS J1813-178 is a fairly complicated ensemble of young massive stars as indicated by several compact HII regions. Figure 5 shows Spitzer Space Telescope data from the GLIMPSE survey at $8 \mu \mathrm{m}$ (Benjamin et al. 2003). As previously mentioned, no dust emission at the position of HESS J1813-178can be found (red contours denote the 1,2, and $3 \sigma$ error contours on the centroid of the VHE $\gamma$-ray emission), however, the peak in the CO-data seems to be correlated to the $8 \mu \mathrm{m}$ data. The fact that most dust in the region that has not yet been blown away by the hot stars might suggest that these stars are still rather young. The total integrated column density determined for a beam size of $2.6^{\prime}$ centred on Galactic longitude 12.8, latitude 0.0 (the closest position to HESS J1813-178 in the NANTEN survey with a grid size of $4^{\prime}$ ) is $\sim 9 \times 10^{22} \mathrm{~cm}^{-2}$, somewhat higher than the value determined by Dickey \& Lockman $(1990)\left(1.6 \times 10^{22} \mathrm{~cm}^{-2}\right)$, but fully consistent with the high absorption density determined from the XMM-Newton data. Integrating the column density only to $4 \mathrm{kpc}$, the proposed location of AXJ1813-178, results in a value of $\sim(0.5-1.) \times 10^{22} \mathrm{~cm}^{-2}$. Comparing the NANTEN values presented here with the values from Dickey \& Lockman (1990) the differences can be attributed to both the difference in material probed (atomic versus molecular hydrogen) and the differing angular resolution of the two surveys. 

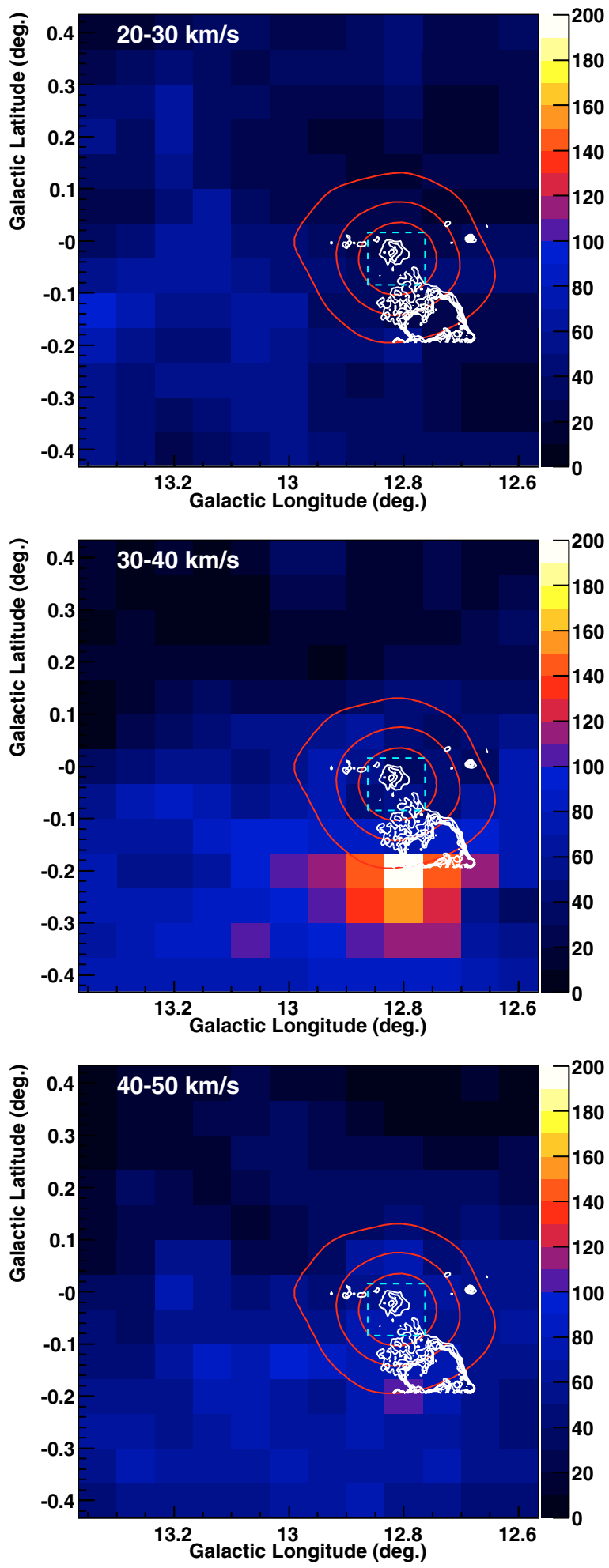

Fig. 4. NANTEN ${ }^{12} \mathrm{CO}(J=1-0)$ data towards the region surrounding HESS J1813-178 for three different velocity ranges. Please note that this figure is in Galactic coordinates. The colour scale shows the $\mathrm{CO}$ emission in units of $\mathrm{K} \mathrm{km} \mathrm{s}^{-1}$, the red contours the HESS VHE $\gamma$ ray excess emission (smoothed with a Gaussian of $0.05^{\circ}$ width and plotted in equidistant excess contours), whereas the white contours show the $20 \mathrm{~cm}$ VLA contours (Brogan et al. 2005). The dashed cyan box indicates the size of the area shown in Fig. 2. The shell-type radio emission at the centre of this region is the SNR G12.82-0.02. As can be seen from this figure, the giant molecular cloud at $30-40 \mathrm{~km} \mathrm{~s}^{-1}$ (corresponding to $\sim 4 \mathrm{kpc}$ ) is not in coincidence with HESS J1813-178, could however be associated with the star-forming region W 33 to the south of HESS J1813-178.

\section{Interpretation of the X-ray emission, connection to other wavebands}

Even though the NANTEN CO-Emission is probably unrelated to HESS J1813-178, it is interesting to calculate an upper limit on the cosmic ray density in the dense molecular cloud given the non-detection of a VHE $\gamma$-ray signal. To calculate the ratio of the cosmic ray density in the cloud $\rho_{\mathrm{CR}, \mathrm{GMC}}$ to the local cosmic ray density $\rho_{\mathrm{CR} \text {,local }}$ the following relation is used:

$$
\rho_{\mathrm{CR}, \mathrm{GMC}} / \rho_{\mathrm{CR}, \mathrm{local}}=\frac{F_{(>1 \mathrm{TeV})} \times D^{2}}{2.85 \times 10^{-13} \times M_{5}\left(\mathrm{kpc}^{2} \mathrm{~cm}^{-2} / \mathrm{s}\right)}
$$

(here $F_{(>1 \mathrm{TeV})}$ is the $\gamma$-ray flux above $1 \mathrm{TeV}$ in units of $\mathrm{cm}^{-2} \mathrm{~s}^{-1}$, $D$ is the distance in kpc, and $M_{5}$ is the mass of the cloud in units of $10^{5}$ solar masses) Aharonian (1991). The factor $2.85 \times$ $10^{-13} \mathrm{kpc}^{2} \mathrm{~cm}^{-2} / \mathrm{s}$ has been derived assuming a CR spectrum with a photon index of 2.6. Using a $2 \sigma$ HESS upper limit of $0.6 \%$ of the Crab flux above $1 \mathrm{TeV}$ (taken from the $10 \mathrm{~h}$ exposure time and the HESS sensitivity of $1 \%$ Crab flux in a $25 \mathrm{~h}$ observation), i.e. $1.1 \times 10^{-13} \mathrm{~cm}^{-2} \mathrm{~s}^{-1}$, a distance of $D=4 \mathrm{kpc}$, and a cloud density of $M_{5}=2.5$, an upper limit for $\rho_{\mathrm{CR}, \mathrm{GMC}}$ of $\sim 3 \times \rho_{\mathrm{CR} \text {,local }}$ can be derived.

Figure 2 shows a comparison between the X-ray and radio emission in the region surrounding HESS J1813-178. As is clearly seen, the radio emission exhibits a shell-like structure whereas the X-ray emission has a compact core with extended emission towards the north-east, a typical morphology for a PWN (see Gaensler \& Slane 2006, for a recent review). The apparent anti-correlation with the radio shell also suggest a confinement of the X-ray emission within the shell, especially since the tail of the X-ray source extends to the NE, where a break in the radio shell is present. The non-detection of a pulsar in reasonably deep radio observations may be due to beaming effects. In the X-ray data presented here the thermal emission from the possible neutron star might be buried underneath the strong non-thermal emission and additionally strongly absorbed by the high column density which strongly affects the detection of X-rays below $\sim 1.5 \mathrm{keV}$. However, to finally confirm this scenario, the pulsar within this nebula would need to be found, either in deep radio or X-ray observations. Alternative strong evidence that AXJ1813-178 is a PWN would be the detection of spectral softening away from the core, a signature of electron cooling which is observed in many PWN, see for example G21.5-0.9 (Slane et al. 2000). The XMM-Newton data do not allow such an effect to be resolved in AX J1813-178. A search for a steepening in the data with respect to the distance of the best fit centroid yielded no conclusive results within statistical errors. Nevertheless, it seems very likely that AX J1813-178 is indeed a PWN, as the positional coincidence of a hard spectrum X-ray source within a radio shell of G12.82-0.02 is otherwise very unlikely. Furthermore, a chance positional coincidence of the VHE $\gamma$-ray source HESS J1813-178 with this composite radio/X-ray object also seems unlikely, and in the following discussion we will assume that the emission seen in these three wavebands originates in a single new composite SNR, similar in its properties to e.g. G $0.9+0.1$ (Aharonian et al. 2005c). However, the situation in the case discussed here is somewhat different, since the VHE $\gamma$-ray source shows extended emission (possibly owing to the smaller distance of HESS J1813-178 ( 4 kpc) in comparison to $\mathrm{G} 0.9+0.1(\sim 8 \mathrm{kpc}))$.

Both the shell of the SNR as well as the newly found PWN candidate AXJ1813-178 are viable $\gamma$-ray emitting objects. To estimate whether the shell of the SNR could be responsible for 


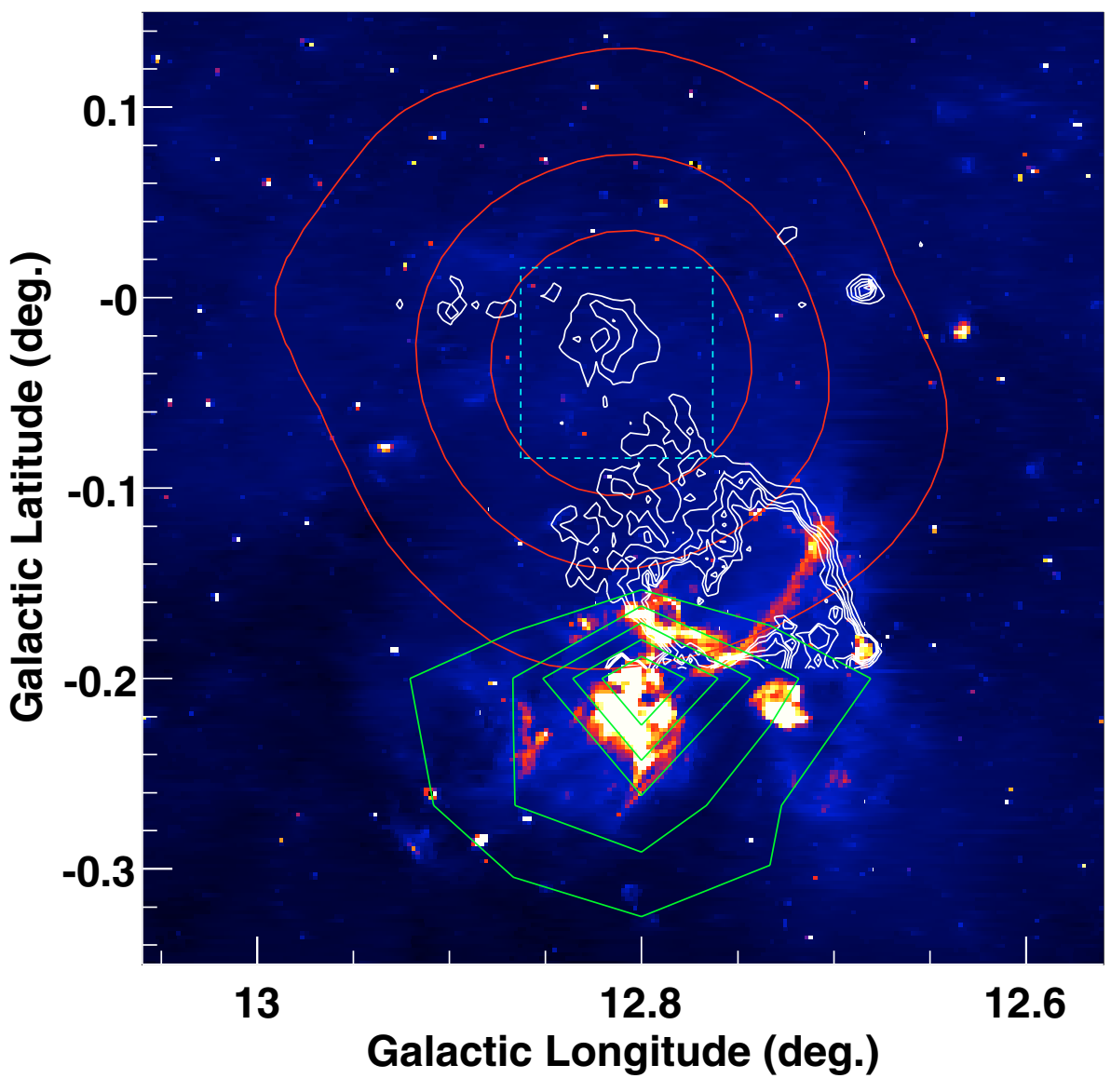

Fig. 5. Spitzer Space telescope data of the GLIMPSE survey at $8 \mu \mathrm{m}$ (Benjamin et al. 2003) shown in Galactic coordinates. The red contours show again the HESS VHE $\gamma$-ray excess emission (smoothed with a Gaussian of $0.05^{\circ}$ width and plotted in equidistant excess contours), whereas the white contours show the $20 \mathrm{~cm}$ VLA contours (Brogan et al. 2005). The green contours show the NANTEN CO-Emission contours at the distance of $4 \mathrm{kpc}$, showing the position of the GMC. The dashed cyan box indicates the size of the area shown in Fig. 2. No dust emission is coincident with the VHE $\gamma$-ray source as reported already by Brogan et al. (2005), supporting the nonthermal nature of the source. The Spitzer and NANTEN data are clearly correlated on this scale. the $\gamma$-ray emission the following prescription has been followed: a background (determined in a ring of 2-4' around the SNR centre) has been subtracted from the central $2^{\prime}$ radio emission, the resulting radio map has been smoothed with a Gaussian kernel of width $r_{\text {smooth }}=1.2^{\prime}$, slices have been fit through the resulting smoothed map in RA- and Dec-Direction with Gaussians to determine $\sigma_{x}$ and $\sigma_{y}$ of the smoothed emission region. Finally the "Gaussian equivalent width" of the SNR has been defined as $\sigma_{\text {SNR }}=\left(\sigma_{x}^{2}+\sigma_{y}^{2}-r_{\text {smooth }}^{2}\right)^{1 / 2}$, yielding $\sigma_{\text {SNR }} \approx 1.8^{\prime}$, compatible with the measured rms extension $2.16^{\prime} \pm 0.36^{\prime}$ of the VHE $\gamma$-ray emission region. The SNR shell seems therefore to be a plausible candidate for the origin of the $\gamma$-ray emission.

As a result, we have to conclude that even with high angular resolution XMM-Newton X-ray observations in which the central X-ray emitting object AXJ1813-178 was resolved, no final distinction can be drawn between the scenario in which the $\gamma$-rays originate in the shell or in the core of G12.82-0.02. The $\mathrm{X}$-ray data described here and previously described radio observations indicate that $\mathrm{G} 12.82-0.02$ is a composite supernova remnant with a bright $\mathrm{X}$-ray core and a radio shell. The size of the gamma-ray source measured by HESS appears to be consistent with an origin of high energy emission in the SNR shell, but a common origin of the X-ray and $\gamma$-ray emission in a central PWN cannot be excluded as a larger spatial extent of the $\gamma$-ray source with respect to the X-ray source could occur in such cases (see for example the case of HESS J1825-137, Aharonian et al. 2006c), due to the energy dependent cooling of electrons. Two distinct scenarios for the origin of the $\mathrm{TeV}$ emission must therefore me considered: 1) as a counterpart to the X-ray emitting core, and 2) as a counterpart to the radio emission of the shell.

Scenario 1) has previously been discussed by Ubertini et al. (2005) and Helfand et al. (2005). Here the situation is revisited in the light of the new XMM-Newton data. Figure 6 (top) shows the spectral energy distribution of G 12.82-0.02. The EGRET upper limit has been derived from the first 5 years of the EGRET mission yielding a flux upper limit of $2.7 \times 10^{-11} \mathrm{erg} \mathrm{cm}^{-2} \mathrm{~s}^{-1}$ above $100 \mathrm{MeV}$. Where angular resolution is sufficient (i.e. in the radio and $<15 \mathrm{keV} \mathrm{X}$-ray bands) the core and shell of the remnant are shown separately. Two synchrotron/Inverse Compton model curves are shown for a population of relativistic electrons in the core. The model was chosen to be time-dependent with constant injection over the lifetime of the source. The key model parameters are the slope of the injection spectrum of electrons $\alpha$ the minimum and maximum energies of the electrons $E_{\min }$ and $E_{\max }$, the magnetic field in the source $B$, and the target radiation field (CMBR, optical or dust photons). In both cases shown, an age of 1000 years is adopted, in-line with the estimates of Brogan et al. (2005) for the age of the remnant: 300-3000 years. The combined $X M M$-Newton/INTEGRAL spectrum indicates that $E_{\max }$ must be rather high: $\sim 10^{15} \mathrm{eV}$. If inverse Compton emission takes place in the Thompson regime then equal keV synchrotron $\mathrm{X}$-ray and VHE $\gamma$-ray IC spectral indices are expected away from any cut-off in the electron spectrum. The softer spectrum measured by HESS therefore suggests that Klein-Nishina (KN) effects may be important in this source. The well-detected X-ray peak determines roughly a convolution of the magnetic field with the square of the maximum electron energy $B \times E_{\max ^{2}}$, whereas the ratio of the total synchrotron to IC emission determines the ratio of $B^{2}$ to the radiation density. Therefore the electron index $\alpha$ is the only free parameter in the modelling when assuming typical radiation fields. A rather soft electron spectrum is needed to match the $\gamma$-ray data, whereas the X-ray data suggests a harder spectrum. The dashed lines in Fig. 6 (top) show a model with $\alpha=2.4, B=4.2 \mu \mathrm{G}, E_{\min }=25 \mathrm{GeV}, E_{\max }=1.5 \mathrm{PeV}$ and 

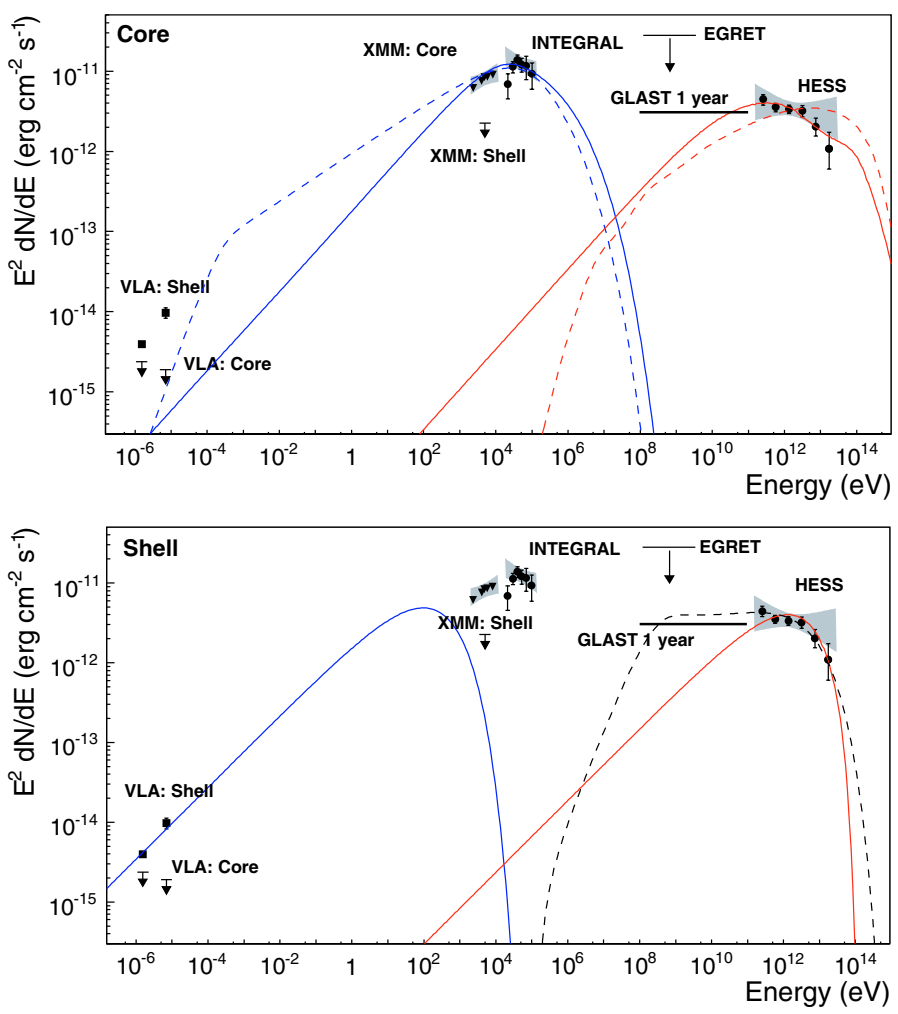

Fig. 6. Spectral energy distribution for HESS J1813-178. The XMM-Newton data $2 \mathrm{keV}$ and $10 \mathrm{keV}$ are shown for an extraction radius of $75^{\prime \prime}$ and are corrected for the absorption. A systematic error band of 0.2 on the spectral index and $20 \%$ on the flux level has been added. For the INTEGRAL points the data published in Ubertini et al. (2005) have been recently reanalysed to determine error bars on the flux points. The flux points shown here are consistent with the published points. The HESS spectrum has been rebinned in comparison to the energy spectrum shown in Aharonian et al. (2006a). Also shown is the 1-year integral sensitivity of the GLAST-LAT above $100 \mathrm{MeV}$ taking into account both the instrumental background and the diffuse $\gamma$-ray emission in the Galactic plane. Top: scenario in which the VHE $\gamma$-ray emission originates in the core of the SNR (i.e. the PWN). Leptonic onezone model (Aharonian \& Atoyan 1999) for two scenarios in which the VHE $\gamma$-ray emitting and the X-ray emitting electrons belong to the same population. The model parameters are specified in the text. Bottom: scenario in which the VHE $\gamma$-ray emission originates in the radio shell of the SNR. Leptonic (solid) and hadronic (dashed) VHE $\gamma$-ray emission scenarios in which the $\gamma$-ray emission originates in the shell of the SNR, i.e. the radio and VHE $\gamma$-ray emitting electrons are connected.

a radiation field with near and far infra-red components each of energy density $1 \mathrm{eV} \mathrm{cm}^{-3}$ in addition to the CMBR. Such a scenario is in marginal agreement with the HESS and X-ray data, but a low energy break or cut-off in the electron spectrum is required to avoid over-producing radio emission in the SNR core. This cutoff could be explained by the termination shock of a PWN, in which the electrons in the lab-system have gained a minimum energy through bulk motion. This minimum energy can be as high as $\sim 100 \mathrm{GeV}$ up to $1 \mathrm{TeV}$, for a typical PWN Lorentz factor between $10^{5}$ and $10^{6}$. The spectral break can be avoided by invoking a strong contribution of scattering by IR/optical radiation fields, where IC scattering transits into the $\mathrm{KN}$ regime and is thus cut off at higher energies, resulting in an apparent steepening of the VHE $\gamma$-ray spectra compared to the X-ray spectra. The solid curves in Fig. 6 (top) show a model with $\alpha=2, B=7.5 \mu \mathrm{G}, E_{\min }=1 \mathrm{MeV}, E_{\max }=1.5 \mathrm{PeV}$ and a radiation field with a very strong NIR component, with energy density $1000 \mathrm{eV} \mathrm{cm}^{-3}$. This radiation field exceeds by a factor of $\approx 1000$ the nominal NIR radiation field at $4 \mathrm{kpc}$ from the Galactic Centre (see e.g. Porter et al. 2006) and is thus somewhat unrealistic. The (possibly) nearby star forming region W 33 may contribute to the radiation density in the vicinity of the source. However, Helfand et al. (2005) have estimated the radiation density in $\mathrm{G} 12.82-0.02$ to be $3-4 \mathrm{eV} \mathrm{cm}^{-3}$, including the contribution of W33. Scenarios with intermediate values provide equally acceptable agreement with the available data. See Hinton \& Aharonian (2007) for details of the calculation methods used here.

A leptonic model, in which the core X-ray and VHE $\gamma$-ray emission are associated, yields an unavoidable $E_{\max }>1 \mathrm{PeV}$, suggesting that HESS J1813-178 is a highly effective accelerator - a Galactic Pevatron. In case of a soft electron injection spectrum $E_{\max }$ could well be higher, since the XMM-NewtonINTEGRAL spectral break could plausibly be a cooling break, rather than represent the end of the electron spectrum, for a somewhat greater pulsar age. If the X-ray and VHE $\gamma$-ray emission are indeed associated (i.e. originate from the same electron population), the PeV maximum energy for the accelerated electrons implies the emission almost certainly has plerionic origin. PWN are certainly capable of accelerating electrons to $\mathrm{PeV}$ energies as e.g. shown by EGRET measurements of the Crab Nebula, showing a synchrotron component extending to $\mathrm{MeV}$ energies (shell type SNRs in the framework of diffusive shock acceleration scheme fall short of $1 \mathrm{PeV}$ for electron acceleration). The total energy injected in the electrons is $6 \times 10^{46} \mathrm{erg}$ for the high-IR case and even $6 \times 10^{47}$ erg for the soft electron spectrum case. For a source of age 1000 years (as suggested by Brogan et al. 2005) these values result in an electron luminosity of $L_{\mathrm{e}} \sim 2 \times 10^{36}-2 \times 10^{37} \mathrm{erg} / \mathrm{s}$. This value can be compared to that of the PWN in the composite SNR G 0.9+0.1 which has a inferred electron luminosity of $L_{\mathrm{e}} \sim 7 \times 10^{37} \mathrm{erg} / \mathrm{s}$ (Hinton \& Aharonian 2007).

A distinct alternative scenario is an origin of the $\gamma$-ray emission in the SNR shell. Two young shell-type SNRs (with estimated ages comparable to that of G 12.82-0.02) are well established VHE $\gamma$-ray sources, namely RXJ1713.7-3946 and RX J0852.0-4622 (known as Vela Junior). Placed at the greater distance of $4 \mathrm{kpc}$, the TeV luminosity of HESS J1813-178 is comparable to that of these two SNRs. Furthermore, the measured size of the TeV emitting region is consistent with an origin in the radio shell. The SNR shell must therefore by considered seriously as an alternative source of the $\mathrm{TeV}$ emission. Figure 6 (bottom) shows two models for a $\gamma$-ray origin in the SNR shell: a) as inverse Compton emission from the electron population responsible for the radio emission (solid line), and b) as the product of the decay of neutral pions produced in hadronic interactions of accelerated protons in or near the SNR shell (dashed line), calculated using the parametrisation of Kelner et al. (2006). In either case, the central X-ray emission must be attributed to a different mechanism, presumably a PWN. The electron model has parameters: $\alpha=2.1, B=5 \mu \mathrm{G}, E_{\min }=$ $1 \mathrm{MeV}, E_{\max }=30 \mathrm{TeV}$ and a nominal $4 \mathrm{kpc}$ radiation field (note that larger values of $E_{\min }$ up to $1 \mathrm{GeV}$ are still compatible with the radio emission). The small value of $E_{\max }=30 \mathrm{TeV}$ is required to avoid producing significant $\mathrm{X}$-ray synchrotron emission in the shell. For values much larger than this, $2 \mathrm{keV}$ emission from the shell should have been seen in this XMM-Newton observation. The proton model has $\alpha=2.1, E_{\min }=1 \mathrm{GeV}$, $E_{\max }=100 \mathrm{TeV}$. To match the TeV flux level in the p-p interaction scenario the product of the total energy in protons and the ambient density must be: $\left(E_{\mathrm{p}} / 10^{50} \mathrm{erg}\right)\left(n / 1 \mathrm{~cm}^{-3}\right) \approx 1$. For 
likely densities greater than $1 \mathrm{~cm}^{-3}$, the required acceleration efficiency is $<10 \%$ for a typical SNR explosion energy of $10^{51} \mathrm{erg}$. In case of a very large local density, Bremsstrahlung effects start to become important and might increase the VHE $\gamma$ ray flux in comparison to the synchrotron X-ray flux. However, as we consider most of the absorption in the X-ray spectrum to be foreground density, the density within the source is likely insufficient for Bremsstrahlung to be the dominant process.

\section{Summary and Conclusion}

Detailed XMM-Newton X-ray and NANTEN ${ }^{12} \mathrm{CO}(J=$ $1-0)$ studies have been performed towards the $\gamma$-ray source HESS J1813-178. The NANTEN data show a giant molecular cloud in the vicinity of HESS J1813-178 that might have played an important role in the evolution of the $\gamma$-ray source. The X-ray data show a resolved non-thermal object in the centre of the shell of the radio SNR, most likely representing synchrotron emission from a PWN. The confirmation of this picture would require the detection of a central pulsar, either in X-ray or in radio observations, or indirectly through the detection of spectral cooling as seen in several other PWN systems. Nevertheless, G 12.820.02 is now tentatively established as a composite SNR through its distinct morphology of a central extended non-thermal object within a radio shell. For the first time, an object of this type has been detected first in VHE $\gamma$-rays and then identified with high-angular resolution radio and X-ray data. This detection shows, that $\gamma$-ray observations are well suited to identify SNR, that are otherwise very hard to detect due to obscuration. No distinction is so far possible between a scenario in which the $\gamma$-rays are emitted from the shell of the SNR, and one in which they are emitted from the central PWN. An upcoming deep Suzaku exposure on HESS J1813-178 will shed more light on the situation in the hard X-ray band and the future GLAST satellite will provide important constraints in the $\mathrm{MeV}-\mathrm{GeV}$ band. If the central X-ray source and the VHE $\gamma$-ray source are indeed connected our modelling of the SED emission suggests that HESS J1813-178 is a Galactic Pevatron.
Acknowledgements. The authors would like to acknowledge the support of their host institutions, and additionally support from the German Ministry for Education and Research (BMBF). Specifically, SF acknowledges support of the Department of energy (DOE). JAH is supported by a UK Particle Physics \& Astronomy Research Council (PPARC) Advanced Fellowship. We would like to thank the whole HESS collaboration for their support. We would also like to thank Y. Uchiyama for providing the analysis of the ASCA data. SF would like to thank C. Brogan and B. Gaensler for useful discussions on this source.

\section{References}

Aharonian, F. A. 1991, Ap\&SS, 180, 305

Aharonian, F. A., \& Atoyan, A. 1999, A\&A, 351, 330

Aharonian, F. A., et al. (HESS Collaboration) 2005a, Science, 307, 1938

Aharonian, F. A., et al. (HESS Collaboration) 2005b, Science, 309, 746

Aharonian, F. A., et al. (HESS Collaboration) 2005c, A\&A, 432, 25

Aharonian, F. A., et al. (HESS Collaboration) 2006a, ApJ, 636, 777

Aharonian, F. A., et al. (HESS Collaboration), 2006b, A\&A, 460, 743

Aharonian, F. A., et al. (HESS Collaboration) 2006c, A\&A, 460, 365

Albert, J. 2006, ApJ, 673, L41

Benjamin, R. A., Churchwell, E., Babler, Brian, L., et al. 2003, PASP, 115, 953

Bertsch, D. L., Dame, T. M., Fichtel, C. E., et al. 1993, ApJ 416, 587

Brand, J., \& Blitz, L. 1993, A\&A, 275, 67

Brogan, C. L., Gaensler, B. M., Gelfand, J. D., et al. 2005, ApJ, 629, L105

Churchwell, E. 1990, A\&ARv, 2, 79

Dickey, J. M., \& Lockman, F. J. 1990, ARA\&A, 28, 215

Funk, S. 2007, Ap\&SS, 309(1-4) [arXiv: astro-ph/0609586]

Goldsmith, P. F., \& Mao, X.-J. 1983, ApJ, 265, 791

Gaensler, B. M., \& Slane, P. O. 2006, ARA\&A, 44, 17

Helfand, D. J., Becker, R. H., \& White, R. L. 2005, ApJL, submitted [arXiv: astro-ph/0505392]

Hinton, J. A., \& Aharonian, F. A. 2007, ApJ, 657, 302

Kelner, S. R., Aharonian, F. A., \& Bugayov, V. V. 2006, Phys. Rev. D, 74, 034018

Landi, R., Bassani, L., Malizia, A., et al. 2006, ApJ, 651, 190

Manchester, R. N. 2001, MNRAS, 328, 17

Mitchell, G. F., Maillard, J.-P., Allen, M., et al. 1990, ApJ, 363, 554

Mizuno, A., \& Fukui, Y. 2004, ASP Conf. Ser., 317, 59

Porter, T. A., Moskalenko, I. V., \& Strong, A. W. 2006, ApJ, 648, L29

Reach, W. T., Rho, J., Tappe, A., et al. 2006, AJ, 131, 1479

Reifenstein, E. C., Wilson, T. L., Burke, B. F., et al. 1970, A\&A, 4, 357

Slane, P., Yang, C., Schulz, N. S., et al. 2000, ApJ, 533, L29

Snowden, S. L, Collier, M. R., \& Kuntz, K. D. 2004, ApJ, 610, 1182

Sugizaki, M., Mitsuda, K., Kaneda, H., et al. 2001, ApJS, 134, 77

Ubertini, P., Bassani, L., Malizia, A., et al. 2005, ApJ, 629, L109

White, R. L., Becker, R. H., \& Helfand, D. J. 2005, AJ 130, 586 